\title{
Sonoporation, a redefined ultrasound modality as therapeutic aid: A review.
}

\author{
Soheyl Sheikh ${ }^{1}$, Shambulingappa Pallagatti ${ }^{1}$, Balwinder Singh ${ }^{2}$, Nidhi Puri ${ }^{2}$, Ravinder Singh ${ }^{3}$, Aman Kalu- \\ cha $^{2}$
}

${ }^{1}$ M.D.S. (Professor), Department of oral medicine and radiology, M.M.College of Dental Sciences and Research, Mullana, Ambala, Haryana(India)

2 Post graduate student, Department of oral medicine and radiology, M.M.College of Dental Sciences and Research, Mullana, Ambala, Haryana(India)

${ }^{3}$ M.D.S. (Sr.lecturer), Department of oral medicine and radiology, M.M.College of Dental Sciences and Research, Mullana, Ambala, Haryana(India)

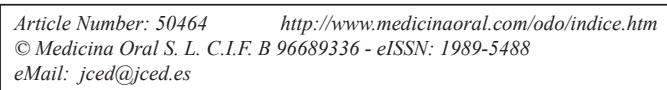

\begin{abstract}
Traditionally a diagnostic modality, ultrasound is emerging as a promising tool for non-invasive therapy, drug delivery, and gene therapy. The ultrasound is a mechanical wave energy generated in a medium as oscillating pressure in space and time at frequencies above $20 \mathrm{kHz}$, beyond the audible range. The ultrasound exposure generates bioeffects resulting in tissue heating, shear stress, and cavitation, which have been exploited for therapeutic applications. Ultrasound cavitation, enhanced by injected micro bubbles, perturbs cell membrane structures to cause sonoporation and increases the permeability to bioactive materials. Ultrasound-mediated gene delivery has been applied to heart, blood vessel, lung, kidney, muscle, head and neck tumour with enhanced gene transfection efficiency. The genes can be incorporated into microbubbles which in turn can target a specific disease site. Local drug delivery ensures sufficient drug concentration at the diseased region while limiting toxicity for healthy tissues. Nevertheless, it has only recently become popular as a technique to enhance drug release from drug delivery systems. This review presents the main findings in the field of sonoporation, namely drug delivery, gene delivery and DNA transfer.
\end{abstract}

Key words: Sonoporation, Gene delivery, Cavitation, Tumor. 


\section{Introduction}

One of the major triumphs in recent times in the field of medicine is Sonoporation.

It is well known that sound is a form of mechanical energy that is propagated from one point to another by the interaction between the neighbouring oscillating particles. The direction of propagation is parallel to the direction of oscillation. Hence, sound is defined as a longitudinal wave.(1) Because its propagation depends entirely on the creation of alternating regions of molecular compression and rarefaction, sound cannot exist in a vacuum. Acoustic waves with frequencies between $20 \mathrm{~Hz}$ and $\sim 20 \mathrm{KHz}$ fall in the audible range. The term ultrasonic refers to sound waves whose frequency is greater than $20 \mathrm{KHz}$. (1)

Its use in technological, navy and aeronautical fields is approximately a century old. Ultrasonic waves are also being used as diagnostic modalities in the medical field since decades but its application in the field of therapeutic medicine and dentistry is new. This new and rapidly developing technology is bringing in a lot of innovations in our field and shows a lot of promise in achieving our aim of advancing clinical care, education, and scientific research.

One such step towards this advancement having its basis in ultrasonic waves is the technique of Sonoporation. The purpose of this paper is to review the literature available on sonoporation as a therapeutic aid, it's possible applications and various studies done in this arena.

\section{Definition}

Sonoporation is defined as the interaction of ultrasound with Ultrasonic contrast agents to temporarily permeabilize the cell membrane allowing for the uptake of various substances such as DNA, drugs, and other therapeutic compounds, from the extracellular environment. (2) This membrane alteration is transient, leaving the compound trapped inside the cell after ultrasound exposure. (3) Sonoporation, unlike other methods of transfection or chemotherapy, combines the capability of enhancing gene and drug transfer with the possibility of restricting this effect to the desired area and the desired time.(4) Thus, after exploring in vitro and in vivo studies on this technique, the therapeutic potential of Sonoporation as a promising drug delivery and gene therapy technique will be reviewed and evaluated in this paper.

\section{Priniciple and Equipment}

The technique of sonoporation is based on ultrasonic waves. The source of these waves is a piezoelectric crystal transducer made of lead-zirconate-titanium or barium titanate. These waves are produced in response to an electrical impulse in the piezoelectric crystal, allowing the conversion of electrical energy into mechanical or vibrational energy. Following the external perturbation, groups of molecules oscillate in phase and transmit their kinetic energy to nearby molecules.

Weiman in 2002 made an apparatus for sonophoretic intradermal delivery of a microparticles suspension containing microencapsulated drugs. This device is a cylindrical container with one end covered with a porous membrane and containing the microparticles suspension. The other end has an ultrasound horn with a tip submerged in the microparticles suspension containing microencapsulated drugs.

The ultrasound radiation is given from ultrasound machine to the microparticles suspension. The ultrasound radiation is applied effectively to generate cavitation bubbles, wherein the cavitation bubbles collapse and transfer their energy into the skin area thus causing the formation of pores in the skin area. (Fig.1)

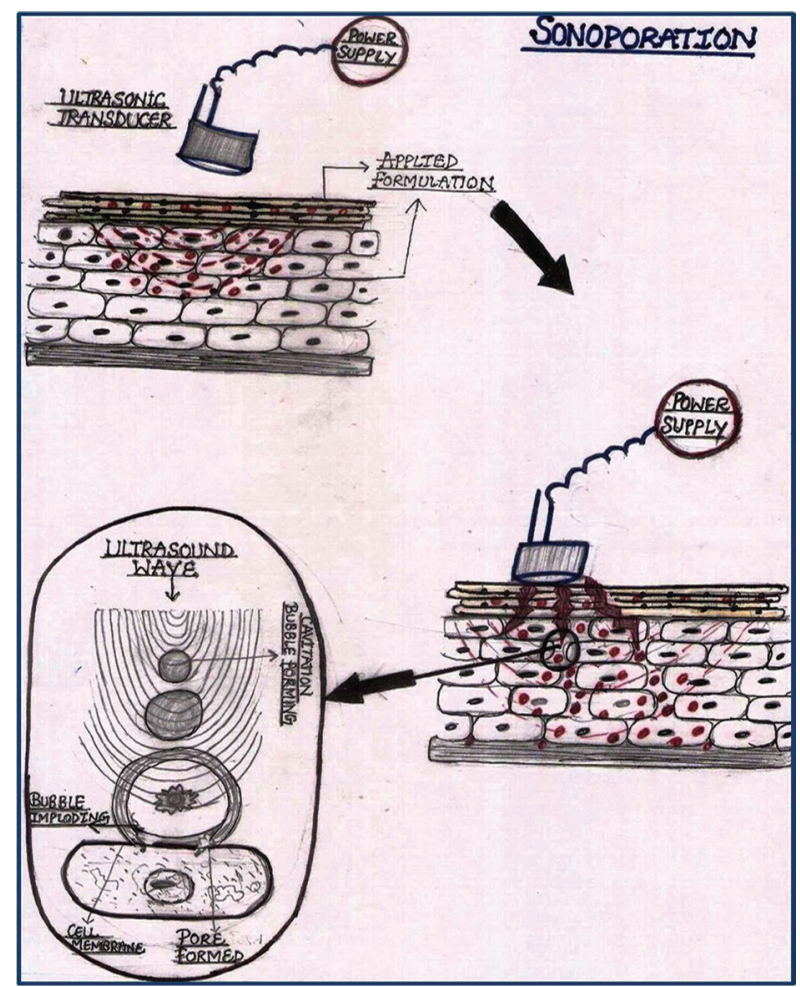

Fig. 1. Mechanism behind Sonoporation.(Hand drawing)

The ultrasound frequency may be $20 \mathrm{KHz}$ and the ultrasound intensity may be in the range of $5 \mathrm{~W} / \mathrm{cm}^{2}$ and 55 $\mathrm{W} / \mathrm{cm}^{2}$. The tip may have a distal end located at a distance from the membrane in the range of 1 millimeter to 10 millimeters. The ultrasound radiation may be continuous or pulsed and it may be applied for a period of time in the range of 30 seconds to 5 minutes, preferably 1 minute for continuous exposure or about 10 to 20 minutes for pulsed exposure with a $5 \%$ duty cycle, respectively. The formed pores may have a diameter in the range of $1 \mathrm{mi}-$ crometer to 100 micrometers.

The ultrasound radiation intensity and distance from the skin area are also effective in generating ultrasonic jets 


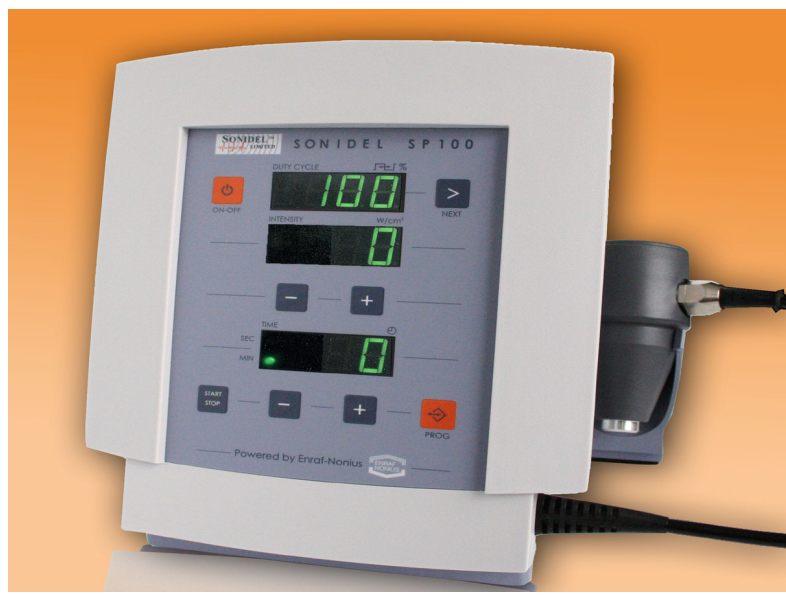

Fig. 2. Sonoporator.

which drive the microparticles suspension containing microencapsulated drugs through the porous membrane and the formed pores into the skin area. Now a day, the sonoporation devices (Fig.2) are commercially available by various companies. (Sonidel Limited MEMS, Texas Scientific Instruments, US). The normal ultrasound device can be used with certain kinds of probes. The probes are based on type of functions like nuclear transfer, cell fusion, tooth germ, tissues etc.

These sound waves can give its effect on formation of pores by possible four ways, as follows(1)-

- Cavitation effects.

- Thermal effects.

- Induction of convective transport.

- Mechanical effects.

Cavitation effect:

The cavitation is the formation of gaseous cavities in medium due to pressure variation in the medium caused by ultrasound waves. This involves both rapid growth and collapse of a bubble (inertial cavitation), or the slow oscillatory motion of a bubble in a medium (stable cavitation).

Collapse of cavitation bubbles releases a shock wave that can cause structural alteration in the surrounding tissue leads to the disordering of the lipid bilayers and formation of aqueous channels in the skin, allowing entry of extracellular agents into the cytoplasm.(1)

\section{Thermal effects:}

Absorption of waves increases temperature of the medium. The tissues with higher absorption coefficients, such as bone will experience severe thermal effects, compared with those with lower absorption coefficient, such as muscle tissues. The increase in the temperature of the medium varies directly with the ultrasound intensity, frequency and exposure time.(1)

Convective transport:

Fluid velocities are generated in porous medium exposed to ultrasound due to interference of the incident and reflected ultrasound waves in the diffusion cell and oscillations of the cavitation bubbles. Fluid velocities

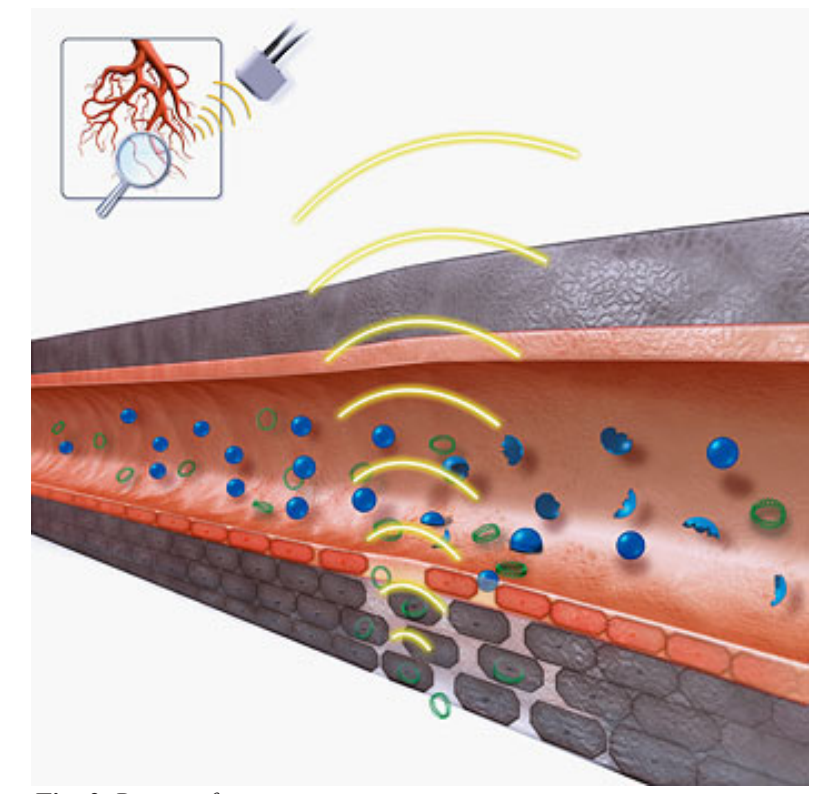

Fig. 3. Basics of sonoporation

may affect transdermal transport by inducing convective transport of the permeate across the skin, especially through hair follicles and sweat ducts.(1)

Mechanical effects:

At frequencies greater than $1 \mathrm{MHz}$, cavitational effects cease. But the other effects like generation of cyclic stresses because of density changes ultimately lead to fatigue of the medium may continue to occur. Lipid bilayers can easily be disordered by these stresses, resulting in an increase in the permeability. Thus cavitation induced lipid bilayer disordering is found to be the most important cause for ultrasonic enhancement of transdermal transport.

\section{Diagnostic ultrasound contrast agents}

The sonophoresis effect will be less effective without the use of ultrasound contrast agents. These agents are suspensions of gas bodies (stabilized micro bubbles), which have been designed for persistence in the circulation and strong echo return for imaging.(3)The interaction of ultrasound pulses with these gas bodies is a form of acoustic cavitation, and they also may act as inertial cavitation nuclei. $(5,6)$

The development of microbubbles as ultrasound contrast agent has opened the way for new and extended application of ultrasound in clinical practise as well as offering new search opportunities.(6)

\section{Applications \\ - Gene delivery \\ Osteoinduction \\ Induction of dental pulp stem cell differentiation into odontoblasts \\ Site specific gene delivery}


DNA transfer

- Local Drug delivery

Targeted Drug delivery

- Tumor cell killing

- Induction of Apoptosis

- Gene transduction

- Mutant construction of bacteria

\section{Gene Delivery}

Gene therapy involves the introduction of a functional gene to replace the activity of a resident defective gene so that biologically active proteins can be synthesized within the cells whose function is to be altered.(7)

Gene therapy was initially thought to be a treatment modality for inherited single gene defects. However it has also found application in acquired diseases. There are about 4000 genetic disorders to be known yet, out of which 700 are in head and neck region alone.(8).Its use is being studied in the treatment of cancer, immunodeficiency diseases, cardiovascular diseases, metabolic disorders, neurological disorders, hormonal disorders and blood factors deficiencies. It is also being developed as a "gene vaccine" against influenza and malaria.(7).

In gene therapy it is very important to develop an effective and safe gene delivery system. Hence in advancement in this arena, Sonoporation induced gene transfer with the help of ultrasound contrast agents was found as an innovative method.

Its applications in field of oral medicine may act as an eye opener for oral physicians who are not familiar with this life altering technique.

Its applications are as follows-

Osteoinduction:

Bone morphogenetic protein is believed to participate in bone healing and regeneration. Gene transfer approach is a promising option for utilizing Bone morphogenic protein.

Osawa Ket al(9) in Japan in 2009 used a plasmid-based human BMP-2 construct (pCAGGS-BMP-2) and examined the induction of bone in the skeletal muscle of mice. The BMP-2 gene was transferred into the skeletal muscle of mice by transcutaneous sonoporation and was assessed by various methods confirming that transcutaneous sonoporation with pCAGGS-BMP-2 caused osteoinduction in the skeletal muscle of mice.

These results suggest the possibility of the effective clinical use of human BMP-2 gene therapy in clinical applications.(9)

Induction of dental pulp stem cell differentiation into odontoblasts:

The long-term goal of dental treatment is to preserve teeth and prolong their function. The dream of the dentist is to restore the lost tooth structure by natural healing is an unfulfilled dream. But this technique has shown some light in this dream which in future will definitely beco- me possible and will possibly allow the natural complete restoration of teeth.

Misako Nakashima et al (10) in 2003 in Japan studied that gene therapy has the potential to induce reparative dentin formation for potential pulp capping. The growth/ differentiation factor 11 (GDF11), a morphogen, could enhance the healing potential of pulp tissue to induce differentiation of pulp stem cells into odontoblasts by sonoporation- mediated gene delivery. Recombinant human GDF11 induced the expression of dentin sialoprotein (Dsp), a differentiation marker for odontoblasts, in mouse dental papilla mesenchyme in organ culture.(10) The in vivo transfer of Gdf11 by sonoporation stimulated the reparative dentin formation during pulpal wound healing in canine teeth.

These results provide the scientific basis and rationale for gene therapy in endodontic treatment and dentistry. Site specific gene delivery:

In gene therapy, it is important to develop an effective and safe gene delivery system. Gene transfer at the required specific site is essential for reducing the side effects, Gene expression was observed only at site of ultrasound exposure.(11) Ultrasound is a very effective modality for drug delivery and gene therapy because energy that is non-invasively transmitted through the skin can be focused deeply into the human body in a specific location and employed to release drugs at that site. Ultrasound-mediated gene delivery has been applied to heart, blood vessel, lung, kidney, muscle, brain, and tumour with enhanced gene transfection efficiency.(12) Moreover, this method was more efficient than the conventional lipofection method.

Shozo sonoda et al (13) in Japan in 2006 significantly tried gene transfer to corneal epithelium and keratocytes mediated by ultrasound with microbubbles and concluded that ultrasound with microbubbles greatly increases gene transfer to in vivo and in vitro corneal cells. This noninvasive gene transfer method may be a useful tool for clinical gene therapy.

DNA transfer:

Delivery of nucleic acid to a target tissue together with a means of facilitating efficient entry of nucleic acid into cell populations of that tissue remain amongst the principle challenges to effective gene therapy in the treatment of a variety of disorders including cancer, cardiovascular disease and inherited immune deficiencies. The development of nonviral gene transfer methods would be a valuable addition to the gene- therapy armamentarium, particularly for localized targeting of specific tissue volume.(4) Ultrasound can produce a variety of non-thermal bioeffects via acoustic cavitation including DNA delivery.

Studies have shown ultrasound enhancement of DNA delivery over direct injection, lipofection, and even over viral transfection. 
Lawrie et al (14) delivered luciferase marker plasmid in porcine vascular smooth muscle cells with naked or lipid-charged DNA with or without ultrasound exposure .The ultrasound application enhanced subsequent luciferase expression by 7.5 times for naked DNA, 2.4 times for lipid-charged DNA in the muscle cells.

The in vivo treatment of cardiovascular problems with help of sonoporation has also been recently reported. The studies done by Taniyama et al. (15) and Yamasaki et al addressed the possibility of preventing vascular proliferation i.e restenosis after angioplasty. These effects are basically seen in those vessels which has been isonated.(16)

\section{Administration of Drugs}

Transdermal drug delivery offers an attractive alternative to the conventional drug delivery methods of oral administration and injection.

The drugs that can be delivered are NSAIDS, anaesthetics, antibiotics, anti-cancer drugs, corticosteroids, insulin and vasodilators.(1) All these drugs have increased penetrance and effect with application of sonoporation technique.

Drugs can be incorporated into microbubbles, which in turn can target a specific disease site using ligands such as the antibody. Drugs can be released ultrasonically from microbubbles that are sufficiently robust to circulate in the blood and retain their cargo of drugs until they enter an insonated volume of tissue. Local drug delivery ensures sufficient drug concentration at the diseased region while limiting toxicity for healthy tissues.(11)

The increase in ultrasound intensity leads to proportional increase in drug transport. The intensity most commonly used was 0.5 to $3.0 \mathrm{~W} / \mathrm{cm} 2$.

Advantages(1)-

1) Allowed strict control of transdermal penetration rates.

2) Less anxiety provoking and less painful then injections.

3) It also allows greater patient satisfaction.

4) Less systemic absorption.

5) The drugs given were less immunological sensitizing.

\section{Tumor Cell Killing}

Ultrasound-mediated destruction of microbubbles has been proposed as an innovative non-invasive drug delivery system for cancer therapy. This will not act only by killing the cells but can give a new favourable look to chemotherapy over other treatment options. The patient will be saved from adverse effects of anticancer drugs which usually act as double edged swords.(17) (Loreto $B$ Feril et al, 2002)

The K. Iwanga et al(18) in 2007 in Japan studied the efficiency of sonoporation toward growth inhibition of human gingival squamous carcinoma cells in vitro and in vivo. The Ca9-22, a human gingival squamous carcinoma cell line was used in this study. Sonoporation was used to deliver bleomycin and transfect a cdtB-expressing plasmid into Ca9-22 cells in vitro and in vivo.

The results showed that tumors nearly disappeared in Ca9-22 cell-implanted treated with bleomycin or cdtBexpressing plasmid during the 4-week experimental period.(18)

Takagi Hironobu et al in 2009 in Japan administered a low dose of bleomycin (BLM) by sonoporation with the anti-EGFR antibody producing a marked growth inhibition of Ca9-22 cells in vitro. (19)

These findings indicate that sonoporation makes it possible to administer drugs into cells more efficiently and specifically, suggesting a novel application for treatment of oral squamous cell carcinoma.

\section{Induction of Apoptosis}

Apoptosis is an organized process of cell death, which occurs naturally for unneeded

cells. This process may be used in future for killing cancerous cells and other cells of benign growths before malignant change or size reduction of the growth before surgeries.

Ashush et al. 2000 (20) and Ando et al in 2006 (21) concluded that exposure of cells to ultrasonic cavitation was shown to induce apoptosis in addition to the conventionally reported instantaneous cell lysis and necrotic disintegration. The induction of apoptosis by $1 \mathrm{MHZ}$ ultrasound exposure has been directly linked to inertial cavitation by its dependence on the presence of an ultrasound contrast agent(22).

\section{Gene Transduction}

Gene transduction technologies are essential tools for understanding of gene functions. Sonoporation is carried out with relatively simple procedures and easily transduces genes into mesenchymal cells without significant damage to target tissues.(23)Therefore, sonoporation is effective for gene transduction to study the molecular mechanisms of morphogenesis.

Sho Ohta et al in 2007 in Japan conducted a study on gene transduction by sonoporation on chick embryos. The survival rates for sonoporated embryos was $96 \%$ after 24-48 hrs and $60 \%$ after 7 days.(23)

\section{Mutant Constuction of Bacteria}

The study of genetic structure growth of microorganisms was often hindered due to lack of genetic tools. The construction of allelic-exchange mutants is essential for understanding the gene functions. Also this technique helped in producing double crossovers in a single step which was not feasible with conventional procedures like electroporation and conjugation. The unique fea- 
tures of one-step double crossovers obtained using intact plasmids signify sonoporation as a potentially powerful genetic tool for streamlining mutant construction.(24) Drawbacks With so many advantages, the negative effects of this technique are minimal. The process may be time consuming. Minor tingling, irritation, and burning have been reported at site of application.(1)

\section{Discussion}

Ultrasound waves were initially only used as a mean of diagnostic aid. It took much longer time to give new direction to these waves to use them as a possible mean of life saving method. The results are creating scientific interest in the therapeutic field of medicine and dentistry. With this technique it will be possible to control major hereditary problems and treat malignancies, thus saving many lives. Sonoporation, just a small vibration force has the power to innovate new treatment methods with wide applications. Though this is still in the infancy stage, it holds a promising and great future prospective.

The sonoporation mediated gene transfer technique was also evaluated for transfection efficiency and safety for gene delivery in comparison with other methods. The results concluded that Sonoporation gene therapy might be the safest technique to be used in actual clinical practice.(4)

Theoretically, sonoporation could help deliver genes with high spatial and temporal specificity. The importance of selective targeting in gene therapy cannot be overemphasized, considering the risks associated with systemic delivery of genetic vectors at high doses. The development of "smart" bubbles that can recognize and attach to specific targets might enhance the spatial resolution even further, providing a real "ZIP code based" delivery of genetic material

Sonoporation has not just enhanced the penetration of drugs at desired amounts and at desired times but also has opened a path for sonophoretic delivery of peptides and protein drugs.(1) Moreover the exact delivery of cytotoxic drugs into specific tumor sites will not just kill the bud in the nip but will also save normal body cells from the toxic side effects of therapeutic drugs.

Thus in coming ages, ultrasound mediated sonoporation is going to gain importance in the therapeutic field of medicine and dentistry along with diagnostic arena and will give new ray of hope of life.

\section{Conclusion}

While in the race of advancing technology, this new avatar of century old ultrasound technique is likely to serve as a boom in the field of diagnosis and therapeutic dentistry. With so many applications with minimal need of equipment, this emerging new form of sound energy will revolutionise many forms of treatment .This possi- bility to transfer therapeutic genes would be a new tool for infancy gene therapy and will take research to new heights. Thus, its simplicity and non invasiveness may provide a new avenue for microinjecting various substances into a wide range of living tissues. With this we can envision a whole gamut of newer technologies and products in the foreseeable future dentistry.

\section{References}

1. Escobar-Chávez JJ, Bonilla-Martínez D, Villegas-González MA, Rodríguez-Cruz IM, Domínguez-Delgado CL. The Use of Sonophoresis in the Administration of Drugs Throughout the Skin. J Pharm Pharmaceut Sci 2009; 12(1):88 - 115.

2. Hallow DM, Mahajan AD, McCutchen ET. Measurement and correlation of acoustic cavitation with cellular bioeffects. Ultrasound in Medicine \& Biology 2006; 32(7):1111-1112.

3. Escoffre J.M, Kaddur K, Rols M.P ,Bouakaz A. In Vitro Gene Transfer by Electrosonoporation. Ultrasound in Medicine \& Biology 2010, 36(10):1746-1755.

4. Miller L.D, Pislaru S.V, Greenleaf J. Sonoporation: Mechanical DNA Delivery by Ultrasonic Cavitation. Somatic Cell and Molecular Genetics 2002; 27(6):115-134.

5. Miller D, Averkiou MA, Brayman AA, Everbach EC, Holland CK, Wible HJ, Wu J. Bioeffects Considerations for Diagnostic Ultrasound Contrast Agents. J Ultrasound Med 2008; 27:611-632.

6. Cosgrove D, Harvey C. Clinical uses of microbubbles in diagnosis and treatment. Med Biol Eng Comp 2010; 47(8):813- 826.

7. Vandana R, Tushar R, Bapna JS. Gene therapy: current status and future. Indian Journal of Pharmacology 1996; 28: 205-219.

8. HC Slavkin. Understanding human genetics .J Am Dent Assoc 1996;127: 266-267.

9. Osawa K, Okubo Y, Nakao K, Koyama N, Bessho K.Osteoinduction by microbubble-enhanced transcutaneous sonoporation of human bone morphogenetic protein-2. The Journal of Gene Medicine 2009; 11(7): 633-641.

10. Nakashima M, Mizunuma K, Murakami T, Akamine A. Induction of Reparative Dentin Formation by Ultrasound-Mediated Gene Delivery of Growth/Differentiation Factor 11. Human gene therapy $2003 ; 14: 591-597$.

11. Suzuki R, Oda Y, Namai E,Takizawa T, Negishi Y, Utoguchi N, Maruyama K. Development of site specific gene delivery system with sonoporation. Yakugaku Zasshi 2008; 128(2):187-92.

12. Liang H,Tang J, Halliwell M. Sonoporation, drug delivery, and gene therapy Proc Inst Mech Eng H. 2010;224(2):343-61.

13. Sonoda S, Tachibana K, Uchino E et al. Gene Transfer to Corneal Epithelium and Keratocytes Mediated by Ultrasound with Microbubbles. Invest Ophthalmol Vis Sci. 2006; 47:558-564.

14. Lawrie A, Brisken AF, Francis SE. Ultrasound enhances reporter gene expression after transfection of vascular cells in vitro. Circulation 1999; 99: 2617-2.

15. Taniyama Y, Tachibana K, Hiraoka K, Namba T, Yamasaki K, HashiyavN, et al. : Local delivery of plasmid DNA into rat carotid artery v using ultrasound. Circulation 2002, 105: 1233-1239.

16. Newman CMH, Bettinger T. Gene therapy progress and prospects: Ultrasound for gene transfer. Gene Therapy 2007; 14, 465-47.

17. Feril BL, Ogawa R, Tachibana K, Kondo T. Optimized ultrasound-mediated gene transfection in cancer cells. Cancer Sci 2006; 97: 1111-1114

18. Iwanaga K, Tominaga K, Yamamoto K, Habu M, Maeda H, Akifusa S, Tsujisawa T, Okinaga T, Fukuda J, Nishihara T. Local delivery system of cytotoxic agents to tumors by focused sonoporation. Cancer Gene Ther. 2007 Apr;14(4):354-63

19. Jingyu C, Xuemo Q, Zhenhua G, Qiao W, Yi T .Targeted drug delivery system for oral cancer therapy using sonoporation. J Oral Pathol Med 2009; 38: 572-579.

20. Ashush, H., L. A. Rozenszajn, et al. (2000). Apoptosis induction of human myeloid leukemic cells by ultrasound exposure." Cancer 
Res 60(4): 1014-20

21. Ando H, Feril L,Kondo T. An echo-contrast agent, Levovist, lowers the ultrasound intensity required to induce apoptosis of human leukemia cells.Cancer letters 2006;242(1):37-45.

22. Miller L, Dou C. Induction of Apoptosis in Sonoporation and Ultrasonic Gene Transfer. Ultrasound Med Biol. 2009 ; 35(1):144-154

23. Ohta S Suzuki K, Ogino Y, Miyagawa S, Murashima A, Matsumaru D, Yamada G. Gene transduction by sonoporation. Develop. Growth Differ. (2008) 50, 517-520.

24. Han W Y, Ikegami A, Chung P, Zhang L, Deng XC. Sonoporation Is an Efficient Tool for Intracellular Fluorescent Dextran Delivery and One-Step Double-Crossover Mutant Construction inFusobacterium nucleatum. Appl. Environ. Microbiol. 2007; 73(11):3677-83 